\title{
DHEA, DHEAS and Prolactin Correlate With Glucose Control Parameters in Women of Fertile Age With Type-1 Diabetes Mellitus
}

\author{
H. KVASNICKOVA ${ }^{1}$, R. HAMPL ${ }^{1}$, K. VONDRA $^{1}$ \\ ${ }^{1}$ Institute of Endocrinology, Prague, Czech Republic
}

Received June 2, 2015

Accepted June 16, 2015

\begin{abstract}
Summary
The aim of our work was to provide data from women of fertile age with type 1 diabetes mellitus about the endogenous androgens and on their relations to the parameters of diabetes control. Forty-two women were examined, they did not use contraceptives for at least three months prior to the examination. A multivariate regression analysis showed that the daily insulin dose, the fasting glycemia and the HbA1c values and patient's age correlated negatively with dehydroepiandrosterone sulfate, dehydroepiandrosterone and prolactin levels. The testosterone/ dehydroepiandrosterone sulfate ratio correlated positively with daily insulin dose and patient's age. In contrast to adrenal androgens the values of other hormones, including total and free testosterone, androstenedione, dihydrotestosterone, estradiol, $\mathrm{LH}, \mathrm{FSH}, 17-\mathrm{OH}-\mathrm{P}$, progesterone and cortisol revealed no significant correlation. To conclude, significant relations between the glucose control parameters and the adrenal androgens and prolactin were demonstrated. These relationships should be considered as an important factor influencing diabetes control so the additional cardiovascular risk in women with DM1.
\end{abstract}

\section{Key words}

Type 1 diabetes mellitus $\bullet$ DHEA/S • Prolactin

\section{Corresponding author}

K. Vondra, Institute of Endocrinology, Národní 8, 11600 Prague 1, Czech Republic. E-mail: kvondra@endo.cz

There are little data in the literature on the relationship between parameters of diabetes control in women with type 1 diabetes mellitus (T1D) at fertile age and endogenous androgens, or other hormones involved in the regulation of reproductive functions. The likely explanation is the fact that a high percentage of these patients use hormonal birth control pills (Sitruk-Ware and Nath 2011). This is why we decided in this study to present our data addressing this problem. We examined 42 women with T1D in fertile age. The average age at the time of examination was $34.0 \pm 7.4$ years, the duration of diabetes $11 \pm 7$ years, BMI $23.7 \pm 3.6$, HbA1c $67 \pm 18 \mathrm{mmol} / \mathrm{mol}$, fasting glycemia $8.1 \pm 3.1 \mathrm{mmol} / \mathrm{l}$, daily dose of insulin $0.59 \pm 0.24 \mathrm{IU} / \mathrm{kg}$. Hormonal characteristics of examined group are given in Table 1. For the study purposes, the patients did not use hormonal birth control pills for at least three months before the examination. All of them were on a long-term intensive insulin therapy. The examination of the thyroid and adrenal gland did not reveal any functional disturbances. The patients signed an informed consent and the study was approved by The Ethical Committee of the Institute of Endocrinology, Prague. Blood was collected in the early follicular phase of the cycle (day 2-5 of cycle) under fasting conditions between 7-9 a.m. Total testosterone (T), sex hormone binding globulin (SHBG), androstenedione (A), dehydroepiandrosterone (DHEA), dehydroepiandro-sterone (DHEAS), dihydrotestosterone (DHT), estradiol, luteinizing hormone (LH), follicle stimulating hormone (FSH), 17 $\alpha$-hydroxyprogesterone (17-OH-P), prolactin (PRL), cortisol, thyroid stimulating hormone (TSH), basal glycemia, glycated hemoglobin (HbA1c) were determined.

Plasma glucose was measured by glucosooxidase methods on Glucose analyser (Beckman, Fullerton, CA); physiological range $3.9-5.5 \mathrm{mmol} / \mathrm{l}$, HbA1c was determined by an immunochemical method using quantitative immunoturbidimetry standardized by 
IFCC (Roche Diagnostics GmbH, Mannheim, Germany), physiological range below $42 \mathrm{mmol} / \mathrm{mol}$ according IFCC. T, A, SHBG, cortisol, DHEA, DHEAS, 17-OH-P and progesterone were measured by radioimmunoassay kits from Immunotech Beckman Coulter Company, Marseille, France. We used the reference range for healthy premenopausal women: for total testosterone $<2.7 \mathrm{nmol} / 1$, for androstenedione $<9.4 \mathrm{nmol} / \mathrm{l}$. Free testosterone was calculated from total testosterone and SHBG according to Vermeulen et al. (1999). SHBG, LH and FSH were determined by radioimmunometric methods (IRMA) using kits from the same purchaser. Estradiol was measured by a Spectria kit from Orion (Oss, The Netherlands). PRL was measured using "Elecsys Prolactin II" heterogenous chemiluminiscent immunoassay by the Analyzer Cobas 6000 from Roche Diagnostics GmbH company. TSH was measured by ECLIA, Roche Diagnostics GmBH, Mannheim, Germany, using Elecsys system 2010, physiological range $0.27-4.20 \mathrm{mIU} / 1$. Their physiological levels of the measured parameters agreed with those reported by the producers (providers).
Table 1. Hormonal characteristics of examined group.

\begin{tabular}{lc}
\hline & mean \pm SD \\
\hline TSH (mIU/l) & $2.9 \pm 0.4$ \\
Prolactin $(\mathrm{ng} / \mathrm{l})$ & $17.1 \pm 9.2$ \\
Cortisol $(\mathrm{nmol} / \mathrm{l})$ & $427.0 \pm 152.8$ \\
Total testosterone (nmol/l) & $2.24 \pm 0.72$ \\
Free testosterone (pmol/l) & $27.14 \pm 10.33$ \\
DHEAS (umol/l) & $5.22 \pm 3.15$ \\
DHEA (nmol/l) & $32.6 \pm 27.8$ \\
Androstenedione (nmol/l) & $5.9 \pm 2.39$ \\
LH $(I U / l)$ & $7.8 \pm 4.9$ \\
FSH $($ IU/l) & $9.6 \pm 6.6$ \\
SHBG (nmol/l) & $67.6 \pm 29.9$ \\
Progesterone (nmol/l) & $17.7 \pm 15.5$ \\
\hline
\end{tabular}

The values were obtained in the early follicular phase of the cycle, only progesterone in the luteal phase of the cycle. TSH, thyroid-stimulating hormone; DHEAS, dehydroepiandrosterone sulfate; DHEA, dehydroepiandrosterone; $\mathrm{LH}$, luteinizing hormone; $\mathrm{FSH}$, follicle-stimulating hormone; SHBG, sex hormone binding protein.

Table 2. Results of multiple regression analysis.

\section{Dependent variables}

\begin{tabular}{|c|c|c|c|c|c|c|c|c|c|c|c|}
\hline \multicolumn{3}{|c|}{ PRL } & \multicolumn{3}{|c|}{ T/DHEAS } & \multicolumn{3}{|c|}{ DHEAS } & \multicolumn{3}{|c|}{ DHEA } \\
\hline $\begin{array}{l}\overline{\bar{d}} \\
\bar{\Xi} \\
\bar{\Xi} \\
\tilde{\Xi} \\
0\end{array}$ & 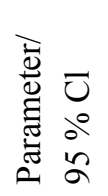 & 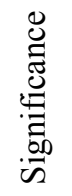 & 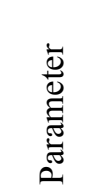 & 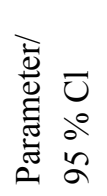 & 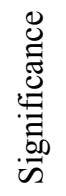 & 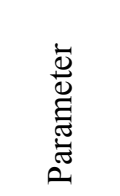 & 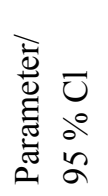 & 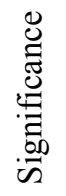 & 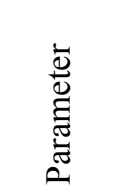 & 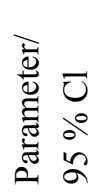 & 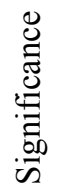 \\
\hline-0.249 & -1.43 & $*$ & 0.277 & 1.76 & $* *$ & -0.323 & -2.91 & $* *$ & -0.277 & -1.33 & $*$ \\
\hline-0.146 & -0.80 & & 0.162 & 1.18 & $*$ & -0.189 & -1.31 & $*$ & -0.162 & -0.76 & \\
\hline-0.168 & -1.64 & $* *$ & 0.187 & 1.48 & $*$ & -0.217 & -1.30 & $*$ & -0.187 & -1.52 & $*$ \\
\hline-0.146 & -1.89 & $* *$ & 0.162 & 0.86 & & -0.189 & -0.94 & & -0.162 & -2.30 & $* *$ \\
\hline-0.204 & -1.50 & $*$ & 0.227 & 0.89 & & -0.265 & -1.16 & $*$ & -0.227 & -1.30 & $*$ \\
\hline
\end{tabular}

PRL, prolactin; T, testosterone; DHEAS, dehydroepiandrosterone sulfate; DHEA, dehydroepiandrosterone; $* p<0.05 ; * * p<0.01$.

An analysis of the relations between clinical characteristics and hormonal findings in the studied group was performed by a multivariate regression. The significant relationships are given in Table 2. PRL correlated negatively with the age of patients $(p<0.05)$, the dose of insulin $(p<0.01)$, HbAlc $(p<0.01)$ and with fasting blood glucose $(\mathrm{p}<0.05)$. DHEAS correlated negatively with the age of patients $(p<0.01)$, with the age when diabetes was diagnosed $(\mathrm{p}<0.05)$, the dose of insulin $(p<0.05)$, and with fasting blood glucose $(p<0.05)$. DHEA correlated negatively with the age of patients $(p<0.05)$, the dose of insulin $(p<0.05), H b A 1 c \quad(p<0.01)$ and with fasting blood glucose $(\mathrm{p}<0.05)$. The ratio T/DHEAS correlated positively with the age of patients $(\mathrm{p}<0.01)$, with the age when diabetes was diagnosed $(p<0.05)$ and with the dose of insulin $(p<0.05)$. Other 
hormones, including total and free testosterone, androstenedione, dihydrotestosterone, estradiol, LH, FSH, 17-OH-P, progesterone and cortisol did not correlate significantly with the variables of diabetic parameters.

The data found in the literature show a higher occurrence of clinical hyperandrogenism in women with T1D, up to $38.8 \%$ in the Spanish population (EscobarMorreale et al. 2000), similar data were provided for Chile (38.1\%) (Codner et al. 2007). In our group, however, there was only three women with clinically manifested hyperandrogenism (Ferriman-Gallwey score over 8), who also had laboratory hyperandrogenemia. Clinical hyperandrogenic manifestations, hirsutism in particular, are less severe in women with T1D (Codner et al. 2006) compared to non-diabetic hyperandrogenic women, and therefore it may be overlooked by a physician.

In the studied group we found $38 \%$ of patients with laboratory hyperandrogenemia. This occurrence is higher than in Codner's cited work (Codner et al. 2006), where laboratory hyperandrogenemia was diagnosed in $23.8 \%$ of women with T1D, or in the group of Spanish T1D cases where it was $19.1 \%$ (Escobar-Morreale et al. 2000). Adrenal androgens DHEA(S) should be examined as part of the differential diagnosis of hyperandrogenic states. Codner et al. 2006 found that DHEAS levels in women with T1D and PCOS are higher compared to women with T1D without proven PCOS.

In analyzing the variability of T1D glucose control parameters we used the method of multivariate regression and thus obtained a more detailed view of the role of the various androgens and other hormones. Above all, the regression analysis demonstrated the key importance of the adrenal androgens DHEA and DHEAS in connection with the variability of the daily dose of insulin, the level of fasting glucose and HbA1c. Both DHEAS and DHEA correlated negatively with the age of patients, the dose of insulin and fasting blood glucose, DHEA more with HbA1c. Similar relationships found Kanazawa et al. (2012) using multiple regression analysis in diabetics type 2 on intensive insulin therapy.
Surprisingly, total testosterone played a role only in relation to DHEA(S). The T/DHEAS ratio was positively linked to the higher daily dosage of insulin showing the T/DHEAS ratio as useful indicator of insulin resistance. In contrast to adrenal androgens, multivariate regression showed the insignificant role of androstenedione, dihydrotestosterone and other analyzed hormones. It is generally accepted that the values of adrenal androgens DHEA(S) physiologically decline with age; this relationship was significantly expressed in our study as well. Multivariate regression analysis showed prolactin values along with $\operatorname{DHEA}(\mathrm{S})$ as the most significant determinants of parameters of glucose control. In interpreting the prolactin - glucose metabolism relationship, it is important to mention experimental findings of Park et al. (2011). He showed that only high prolactin levels induce glucose intolerance, hyperinsulinemia and insulin resistance, which is in full agreement with clinical experience in patients suffering from hyperprolactinemia (Berinder et al. 2011, Atmaca et al. 2013). Moderately increased prolactin levels were in Park's study associated with improvement of insulin secretion and sensitivity. In accordance with this observation are correlations between slightly increasing levels of prolactin and improving diabetes control parameters revealed in our study.

In summary, significant relations between parameters of glucose control and the levels of DHEA(S) and prolactin were demonstrated. Moreover, the T/DHEAS ratio was positively associated with the daily dose of insulin and vice versa. These relationships should be considered as an important factor influencing diabetes control so the additional cardiovascular risk in women with DM1 (García-Romero and Escobar-Morreale 2006, Amato et al. 2014).

\section{Conflict of Interest}

There is no conflict of interest.

\section{Acknowledgements}

Supported by MH CZ - DRO (Institute of Endocrinology - EÚ, 00023761).

\section{References}

AMATO MC, GUARNOTTA V, CIRESI A, MODICA R, PANTÒ F, GIORDANO C: No phenotypic differences for polycystic ovary syndrome (PCOS) between women with and without type 1 diabetes mellitus. Clin Endocrinol Metab 9: 203-211, 2014. 
ATMACA A, BILGICI B, ECEMIS GC, TUNCEL OK: Evaluation of body weight, insulin resistance, leptin and adiponectin levels in premenopausal women with hyperprolactinemia. Endocrine 44: 756-612, 2013.

BERINDER K, NYSTRÖM T, HÖYBYE C, HALL K, HULTING AL: Insulin sensitivity and lipid profile in prolactinoma patients before and after normalization of prolactin by dopamine agonist therapy. Pituitary 14: 199-207, 2011.

CODNER E, SOTO N, LOPEZ P, TREJO L, AVILA A, EYZAGUIRRE FC, INIGUEZ G, CASSORLA F: Diagnostic criteria for polycystic ovary syndrome and ovarian morphology in women with type 1 diabetes mellitus. $J$ Clin Endocrinol Metab 91: 2250-2256, 2006.

CODNER E, INIGUEZ G, VILLARROEL C, LOPEZ P, SOTO N, SIR-PETERMANN T, CASSORLA F, REY RA: Hormonal profile in women with polycystic ovarian syndrome with or without type 1 diabetes mellitus. $J$ Clin Endocrinol Metab 92: 4742-4746, 2007.

CODNER E, ESCOBAR-MORREALE H: Clinical review: Hyperandrogenism and polycystic ovary syndrome in women with type 1 diabetes mellitus J Clin Endocrinol Metab 92: 1209-1216, 2007.

ESCOBAR-MORREALE HF, ROLDÁN B, BARRIO R, ALONSO M, SANCHO J, DE LA CALLE H, GARCÍAROBLES R: High prevalence of the polycystic ovary syndrome and hirsutism in women with type 1 diabetes mellitus. J Clin Endocrinol Metab 85: 4182-4187, 2000.

GARCÍA-ROMERO G, ESCOBAR-MORREALE HF: Hyperandrogenism, insulin resistance and hyperinsulinemia as cardiovascular risk factors in diabetes mellitus Curr Diabetes Rev 2: 39-49, 2006.

KANAZAWA I, YAMAGUCHI T, SUGIMOTO T: Effects of intensive glycemic control on serum levels of insulinlike growth factor-I and dehydroepiandrosterone in Type 2 diabetes mellitus. J Endocrinol Invest 35: 469-472, 2012.

PARK S, KIM DA S, DAILY JW, KIM SH: Serum prolactin concentrations determine whether they improve or impair beta-cell function and insulin sensitivity in diabetic rats. Diabetes Metab Res Rev 27: 564-574, 2011.

SITRUK-WARE R, NATH A: Metabolic effects of contraceptive steroids. Rev Endocr Metab Disord 12: 63-75, 2011.

VERMEULEN A, VERDONCK L, KAUFMAN JM: A critical evaluation of simple methods for the estimation of free testosterone in serum. J Clin Endocrinol Metab 84: 3666-3672, 1999. 